\title{
APPLICATION OF THE DUAL-PROCESS METHOD TO THE STUDY OF A CERTAIN SINGULAR DIFFUSION
}

BY

DAVID WILLIAMS

\begin{abstract}
This paper should be regarded as a sequel to a paper by Holley, Stroock and the author. Its primary purpose is to provide further illustration of the application of the dual-process method. The main result is that if $d>2$ and $\varphi$ is the characteristic function of an aperiodic random walk on $\mathrm{Z}^{d}$, then there is precisely one Feller semigroup on the $d$-dimensional torus with generator extending $A=\{1-\varphi(\theta)\} \Delta$. A necessary and sufficient condition for the associated Feller process to leave the singular point 0 is determined. This condition provides a criterion for uniqueness in law of a stochastic differential equation which is naturally associated with the process.
\end{abstract}

\section{Introduction.}

1.1. This paper should be regarded as a sequel to [4]; anyone reading it must realise that ideas of Holley and Stroock run throughout. The notation of [4] is used here.

The 1-dimensional story, which has many interesting features which have no counterpart in dimension $d \geqslant 2$, is told in full in [4]. We therefore assume throughout this paper that $d \geqslant 2$.

I wish to thank Professor D. W. Stroock and the referee for pointing out some errors in an earlier version of this paper.

1.2. Let $T^{d}$ be the $d$-dimensional torus. Think of $T^{d}$ as $[-\pi, \pi]^{d}$ with the obvious identification. For $\theta=\left(\theta_{1}, \theta_{2}, \ldots, \theta_{d}\right) \in T^{d}$ (with $\left|\theta_{k}\right|<\pi, \forall k$ ), put

$$
|\theta| \equiv\left\{\sum \theta_{k}^{2}\right\}^{1 / 2}
$$

(The symbol "三" signifies "is defined to be equal to".) The character group of $T^{d}$ is, of course, the $d$-dimensional integer lattice $\mathbf{Z}^{d}$. Write

$$
e_{\mathbf{n}}(\boldsymbol{\theta})=e_{\theta}(\mathbf{n})=e^{i \mathrm{n} \cdot \boldsymbol{\theta}} \quad\left(\mathrm{n} \in \mathbf{Z}^{d}, \boldsymbol{\theta} \in T^{d}\right) .
$$

Let $\left\{p_{\mathbf{n}}: \mathbf{n} \in \mathbf{Z}^{d}\right\}$ be a symmetric probability distribution on $\mathbf{Z}^{d}$ so that (with the obvious parameter ranges)

$$
p_{\mathrm{n}}=p_{-\mathrm{n}} \geqslant 0 ; \quad \sum p_{\mathrm{n}}=1 .
$$

Received by the editors August 6, 1976.

AMS (MOS) subject classifications (1970). Primary 60J35, 60J60; Secondary 60H10.

Key words and phrases. Dual process, diffusion, Markov chain, Feller property, Bochner map, stochastic differential equation, Girsanov's example.

- American Mathematical Society 1978 
Let $\varphi$ be the characteristic function of $\left\{p_{\mathbf{n}}\right\}$ :

$$
\varphi(\theta) \equiv \sum p_{\mathrm{n}} e_{\mathrm{n}}(\theta) \quad\left(\theta \in T^{d}\right) .
$$

Make the aperiodicity assumption: $\varphi(\theta)=1 \Rightarrow \theta=0$. Put

$$
\mathcal{L} \equiv[1-\varphi(\theta)] \Delta, \quad \mathscr{D}(\mathfrak{L}) \equiv C^{2}\left(T^{d}\right),
$$

where $\Delta$ is the Laplacian on $T^{d}$.

By a Feller version of $e^{t \mathfrak{l}}$, we mean a positive contraction semigroup $\left\{T_{t}\right.$ : $t>0$ \} on $C\left(T^{d}\right)$ such that

$$
T_{\imath} f-f=\int_{0}^{t} T_{s} \mathcal{L} f d s, \quad \forall f \in C^{2}\left(T^{d}\right) .
$$

Such a semigroup is necessarily strongly continuous on $C\left(T^{d}\right)$ and the point of (1) is that the infinitesimal generator of $\left\{T_{t}\right\}$ is an extension of $\mathcal{L}$.

THEOREM 1. There is precisely one Feller version of $e^{t \mathfrak{l}}$. We shall denote this unique Feller version by $\left\{T_{t}^{F}\right\}$.

[The analogue of Theorem 1 is false in the case when $d=1$; in that case, infinitely many Feller versions of $e^{t \mathfrak{L}}$ exist for certain $\mathcal{L}$. See [4].]

Let $\left\{\theta^{F}(t)\right\}$ be a (continuous, strong Markov) diffusion process on $T^{d}$ which has $\left\{T_{t}^{F}\right\}$ as its transition semigroup. Away from $0,\left\{\theta_{t}^{F}\right\}$ behaves as a "Brownian motion run at rate $2[1-\varphi \circ \theta(t)]$ ". Thus, if started away from 0 , $\left\{\theta^{F}(t)\right\}$ will never hit 0 . We see that 0 is either an absorbing point for $\left\{\theta^{F}(t)\right\}$ or else is a "pure entrance boundary" point for $\left\{\theta^{F}(t)\right\}$ which is left immediately never to be revisited. (The strong Markov property rules out the possibility that $\left\{\theta^{F}(t)\right\}$ stays at 0 for an exponential time and then leaves continuously.)

Set

$$
\begin{aligned}
I & \equiv \int_{T^{2}}|(\log |\theta|)|[1-\varphi(\theta)]^{-1} d \theta \quad \text { if } d=2, \\
& \equiv \int_{T^{d}}|\theta|^{2-d}[1-\varphi(\theta)]^{-1} d \theta \quad \text { if } d>3 ;
\end{aligned}
$$

here $d \theta$ denotes the Haar measure on $T^{d}$.

TheOREM 2. The point 0 is absorbing for $\left\{\theta^{F}(t)\right\}$ if and only if $I=\infty$.

We may translate Theorem 2 and the results established during its proof into the language of stochastic differential equations. To do this, we need to use the terminology of the important paper [7] by Yamada and Watanabe. Consider the stochastic differential equation

$$
d \mathbf{x}_{t}=2^{1 / 2}\left[1-\varphi\left(\mathbf{x}_{t}\right)\right]^{1 / 2} d \mathbf{b}_{t}, \quad \mathbf{x}_{0}=0,
$$

where $\varphi$ is now considered lifted from $T^{d}$ to $\mathbf{R}^{d}$. As in [7], we understand by a 
solution of (2) a set-up $\left(\Omega, \mathscr{F}, P ; \mathscr{F}_{t}\right)$ and a pair $\left(\left\{\mathbf{x}_{t}\right\},\left\{\mathbf{b}_{t}\right\}\right)$ of continuous $\left\{\mathscr{F}_{t}\right\}$ adapted processes such that $\left\{b_{t}\right\}$ is an $\mathbf{R}^{d}$-valued Brownian motion relative to $\left\{\mathscr{F}_{t}\right\}$ and that (2) holds in the usual Itô sense. Note especially that a solution is not assumed to be 'strong' in the sense that (for each $t$ ) $\mathbf{x}_{t}$ is measurable on the $\sigma$-algebra generated by $\left\{\mathbf{b}_{s}: s \leqslant t\right\}$.

We shall call a solution $\left(\Omega, \mathscr{F}, P ; \mathscr{F}_{t},\left\{\mathbf{x}_{t}\right\},\left\{\mathbf{b}_{t}\right\}\right)$ of (2) trivial if $P\left\{\mathbf{x}_{t}=\mathbf{0}\right.$, $\forall t\}=1$.

THEOREM 3. A nontrivial solution of equation (2) exists if and only if $I<\infty$.

Because Yamada and Watanabe already give very tight generalised Hölder conditions for uniqueness of solutions to stochastic differential equations, the only real interest of Theorem 3 lies in the fact that $1-\varphi(\mathbf{x})$ can have different asymptotic behaviour as $\mathbf{x} \rightarrow \mathbf{0}$ from different directions. Against this must be weighed the facts that $\varphi$ is positive-definite and that $1-\varphi(\mathbf{x})$ is bounded below by a multiple of $|\mathbf{x}|^{2}$ near 0. See Proposition 7.5 in Spitzer [5].

Here is a rather crude illustrative example. It only emphasizes once again that, for singular points in dimension $d \geqslant 2$, one cannot improve very significantly on Itô's Lipschitz condition for uniqueness.

EXAMPLE. We apply Theorem 3 to an equation reminiscent of Girsanov's famous example in [2]. Suppose that $0<\alpha_{k} \leqslant 1$ for $k=1,2$. Then the equation

$$
d \mathbf{x}=\left\{\left|x_{1}\right|^{2 \alpha_{1}}+\left|x_{2}\right|^{2 \alpha_{2}}\right\}^{1 / 2} d \mathbf{b}, \quad \mathbf{x}_{0}=\mathbf{0},
$$

has a nontrivial solution unless $\alpha_{1}=\alpha_{2}=1$. (Inclusion of logarithmic terms could enliven the statement of the example, but the integrals become complicated. The points raised by Theorems 1-3 which are worth pursuing lie in other directions.)

1.3. The whole point of this paper is to show how results like Theorems 1,2 and 3 may be proved by the dual-process method developed in [3], [4].

It is easily verified that

$$
\mathcal{L} e_{\mathbf{m}}(\boldsymbol{\theta})=\hat{\mathscr{L}} e_{\theta}(\mathbf{m}) \equiv \sum_{\mathbf{n}} \hat{\mathcal{L}}(\mathbf{m}, \mathbf{n}) e_{\theta}(\mathbf{n}),
$$

where

$$
\hat{\mathfrak{L}}(\mathbf{m}, \mathbf{n}) \equiv|\mathbf{m}|^{2} \mathscr{Q}(\mathbf{m}, \mathbf{n}),
$$

$\mathbb{Q}$ being the 'generator' matrix of the $\left\{p_{\mathbf{n}}\right\}$ random walk:

$$
\mathcal{Q}(\mathbf{m}, \mathbf{n}) \equiv p_{\mathbf{n}-\mathbf{m}}-\delta_{\mathbf{m}, \mathbf{n}} .
$$

Note that $\hat{\mathcal{L}}$ is a $Q$-matrix in the sense of chain theory. Let $\left\{\hat{T}_{t}^{\min }\right\}$ be the minimal transition function with $Q$-matrix $\hat{\mathcal{L}}$. The associated minimal chain $\left\{\mathbf{n}^{\min }(t)\right\}=\left\{\mathbf{n}_{t}^{\min }\right\}$ is the $\left\{p_{\mathbf{n}}\right\}$ random walk "run at rate $\left|\mathbf{n}_{t}^{\min }\right|^{2}$ and killed at 
the time $\zeta$ when $\mathbf{n}_{t}^{\min }$ first reaches $\infty "$. Note that $\mathbf{0}$ is an absorbing point for this chain.

Suppose that $\left\{T_{t}\right\}$ is a Feller version of $e^{t \mathfrak{l}}$. Then, for each $t, T_{t}: C \rightarrow C$, where $C$ denotes $C\left(T^{d}\right)$. Hence $T_{t}^{*}: M \rightarrow M$ where $M=M\left(T^{d}\right)=C^{*}$ is the Banach space of Borel signed measures on $T^{d}$ with finite total-variation norm; further, $T_{i}^{*}: M^{+} \rightarrow M^{+}$, where $M^{+}$denotes the set of positive measures in $M$. For $\mu \in M$, write

$$
\hat{\mu}(\mathbf{n}) \equiv \int_{T^{d}} e_{\mathbf{n}}(\theta) \mu(d \theta),
$$

and use the notation $\left(T_{t}^{*} \mu\right)^{\wedge}$ for $\hat{\nu}$ where $\nu=T_{t}^{*} \mu$.

Theorem 1 hinges on the fact that for each $t$, the operator $T_{t}^{F}$ is completely characterised by the duality relation:

$$
\left(T_{t}^{F *} \mu\right)^{\wedge}=\hat{T}_{t}^{\min \hat{\mu}} \quad(\forall \mu \in M) ;
$$

we say (see [4]) that $\left\{\hat{T}_{t}^{\min }\right\}$ is the Bochner dual of $\left\{T_{t}^{F}\right\}$. We shall use a perturbation argument to show that (for fixed $t$ ) $\hat{T}_{t}^{\min }$ is a Bochner map, that is, $\hat{T}_{t}^{\min }$ maps positive-definite functions to positive-definite functions. We can then use (5) to define the (positive) map $T_{t}^{F}: C \rightarrow C$.

\section{Proof of Theorem 1.}

2.1. Existence of $\left\{T_{t}^{F}\right\}$. For $\varepsilon>0$, let $g^{e}$ be the strictly elliptic operator defined by

$$
\mathcal{G}^{e} \equiv \mathcal{L}+\varepsilon \Delta \text { with } \mathscr{D}\left(\mathcal{S}^{e}\right) \equiv C^{2}\left(T^{d}\right) .
$$

Let $\mathrm{B}$ be a Brownian motion on $T^{d}$. Set

$$
\begin{aligned}
\tau^{e}(t) & \equiv \frac{1}{2} \int_{0}^{t}\left[1+\varepsilon-\varphi\left(\mathbf{B}_{s}\right)\right]^{-1} d s, \\
\gamma^{\varepsilon}(t) & \equiv \inf \left\{s: \tau^{\varepsilon}(s)>t\right\} .
\end{aligned}
$$

Then $\left\{\boldsymbol{\theta}^{\varepsilon}(t)\right\} \equiv\left\{\mathbf{B} \circ \gamma^{\varepsilon}(t)\right\}$ is a diffusion process on $T^{d}$. By Hunt-LampertiYang weak-convergence methods [8], it is easy to show that the transition semigroup $\left\{U_{t}^{e}\right\}$ of $\left\{\theta^{\varepsilon}(t)\right\}$ is Feller. By a well-known Dynkin-Volkonskii result, the infinitesimal generator of $\left\{U^{e}(t)\right\}$ extends $\mathcal{G}^{e}$. That $\left\{U_{t}^{e}\right\}$ is the unique Feller version of $\exp \left(t \mathcal{G}^{e}\right)$ either may be read off from deep results of Stroock and Varadhan [6] and Yang [8] or may be proved by the dual-process method of [4]. The idea of the latter method is that any Feller version $\left\{U_{t}^{e}\right\}$ of $\exp \left(t \mathcal{G}^{e}\right)$ must be characterised by the fact that

$$
\left(U_{t}^{\varepsilon *} \mu\right)^{\wedge}(\mathbf{n})=\hat{E}_{n}\left[\hat{\mu} \circ \mathbf{n}^{\min }(t) \exp \left\{-\int_{0}^{t} \varepsilon\left|\mathbf{n}^{\min }(s)\right|^{2} d s\right\} ; \zeta>t\right],
$$

where $\hat{E}_{\mathbf{n}}$ denotes expectation corresponding to starting-position $\mathbf{n}$ for the minimal $\hat{\mathrm{C}}$ chain $\left\{\mathbf{n}^{\min }(t)\right\}$. (Recall that $\zeta$ is the explosion time for $\left\{\mathbf{n}^{\min }(t)\right\}$.) 
See [4] for a proof of (6), but note that (6) is "algebraically plausible" because of the relation

$$
\mathcal{G}^{\varepsilon} e_{\mathbf{n}}(\boldsymbol{\theta})=\left[\hat{\mathcal{L}}-\varepsilon|\mathbf{n}|^{2}\right] e_{\theta}(\mathbf{n})
$$

and the Feynman-Kac formula.

The dominated-convergence theorem shows that as $\varepsilon \downarrow 0$, the right-hand side of equation (6) converges to $\hat{T}_{t}^{\min } \hat{\mu}(\mathrm{n})$. Hence, for $\mu \in M\left(T^{d}\right)$, the weak* limit

$$
T_{t}^{F *} \mu \equiv \underset{e \downarrow 0}{*}-\lim U_{t}^{e *} \mu
$$

exists and

$$
\left(T_{t}^{F *} \mu\right)^{\wedge}=T_{t}^{\min } \hat{\mu} .
$$

From (7), each $T_{t}^{F *}(t>0)$ maps probability measures to probability measures. Suppose that $\left\{\mu_{k}\right\}_{1}^{\infty}$ is a sequence of elements of $M\left(T^{d}\right)$ such that $\mu_{k} \rightarrow \mu \in M$ in the weak* topology. Then, by the uniform-boundedness principle,

$$
\left|\mu_{k}(\mathbf{n})\right| \leqslant\left\|\mu_{k}\right\| \leqslant \sup _{k}\left\|\mu_{k}\right\|<\infty .
$$

Hence, by the dominated-convergence theorem, as $k \rightarrow \infty$,

$$
\begin{aligned}
\left(T_{t}^{F *} \mu_{k}\right)^{\wedge}(\mathbf{m}) & =\hat{T}_{t}^{\min } \hat{\mu}_{k}(\mathbf{m})=\sum_{\mathbf{n}} \hat{T}_{t}^{\min }(\mathbf{m}, \mathbf{n}) \hat{\mu}_{k}(\mathbf{n}) \\
& \rightarrow \sum \hat{T}_{t}^{\min }(\mathbf{m}, \mathbf{n}) \hat{\mu}(\mathbf{n})=\hat{T}_{t}^{\min } \hat{\mu}(\mathbf{m})=\left(T_{t}^{F *} \mu\right)^{\wedge}(\mathbf{m}),
\end{aligned}
$$

whence $T_{t}^{F *} \mu_{k} \rightarrow T_{t}^{F *} \mu$ in the weak ${ }^{*}$ sense.

Now put

$$
T_{t}^{F} f(\boldsymbol{\theta}) \equiv \int_{T^{d}} f(\boldsymbol{\alpha})\left(T_{t}^{F *} \delta_{\theta}\right)(d \boldsymbol{\alpha}) \quad\left(\forall f \in C\left(T^{d}\right)\right),
$$

where $\delta_{\theta}$ denotes the unit mass at $\theta$. Then $T_{t}^{F}$ is easily seen to be a positive map from $C\left(T^{d}\right)$ to $C\left(T^{d}\right)$. See [4], where it is also shown that property (1) will follow for $\left\{T_{t}^{F}\right\}$ once we establish that

$$
\hat{T}_{t}^{\min } \hat{\mu}(\mathbf{n})-\hat{\mu}(\mathbf{n})=\int_{0}^{t} \hat{\mathcal{L}} \hat{T}^{\min } \hat{\mu}(\mathbf{n}) d s \quad\left(\forall \mathbf{n} \in \mathbf{Z}^{d}\right),
$$

where $\mu \in M\left(T^{d}\right)$. However, it is an old result from chain theory that (9) will hold if $\hat{\mu}$ is an arbitrary element of $B\left(\mathbf{Z}^{d}\right)$.

We have now completed the proof of the existence of a Feller version $\left\{T_{t}^{F}\right\}$ of $e^{t \mathfrak{L}}$ characterised by equation (8). Clearly, this 'existence' proof (unlike the 'uniqueness' proof to follow) works for $d=1$ too.

2.2. Uniqueness of $\left\{T_{t}^{F}\right\}$. Let $\left\{T_{t}\right\}$ be any Feller version of $e^{t \mathfrak{l}}$. For $\lambda>0$ and $f \in C \equiv C\left(T^{d}\right)$, put 


$$
R_{\lambda} f(\theta) \equiv \int_{[0, \infty)} e^{-\lambda t} T, f(\theta) d t \quad\left(\theta \in T^{d}\right) .
$$

Then $R_{\lambda}: C \rightarrow C$ and, from (1),

$$
R_{\lambda}(\lambda-\mathcal{L}) f=f \quad\left(\forall f \in C^{2}\left(T^{d}\right)\right) .
$$

Put $u_{\lambda, \theta}(\mathrm{n}) \equiv R_{\lambda} e_{\mathrm{n}}(\theta)$. Then, with one use of Fubini's theorem,

$$
\begin{aligned}
\lambda u_{\lambda, \theta}(\mathbf{n})-e_{\mathbf{n}}(\boldsymbol{\theta}) & =R_{\lambda} \mathscr{L} e_{\mathbf{n}}(\boldsymbol{\theta})=|\mathbf{n}|^{2} R_{\lambda} \mathscr{Q} e_{\theta}(\mathbf{n}) \\
& =|\mathbf{n}|^{2} \mathscr{Q} R_{\lambda} e_{\mathbf{n}}(\boldsymbol{\theta})=\hat{\mathcal{L}} u_{\lambda, \theta}(\mathbf{n}),
\end{aligned}
$$

whence

$$
(\lambda-\hat{\mathfrak{L}}) u_{\lambda, \theta}(\mathbf{n})=e_{\theta}(\mathbf{n}) .
$$

Amply enough theory is known [6], [8] to enable one to prove rigorously that a diffusion process $\left\{\boldsymbol{\theta}_{t}\right\}$ with transition semigroup $\left\{T_{t}\right\}$ will, while away from 0 , behave as a Brownian motion run at rate $2\left[1-\varphi\left(\theta_{t}\right)\right]$. Hence, using the hypothesis that the dimension $d \geqslant 2$, we deduce that if $\left\{\boldsymbol{\theta}_{t}\right\}$ is started at a point $\theta \neq 0$, then $\left\{\boldsymbol{\theta}_{t}\right\}$ will never hit $\mathbf{0}$. Furthermore, again using $d \geqslant 2$, it is clear from the time-substitution argument that for $\theta \neq 0$, the kernel $R_{\lambda}(\theta, \cdot)$ of $R_{\lambda}$ is absolutely continuous with respect to the Haar measure. Hence, by the Riemann-Lebesgue lemma,

$$
\lim _{\mathbf{n} \rightarrow \infty} u_{\lambda, \theta}(\mathbf{n})=0 \text { for } \theta \neq 0 .
$$

By Feller's boundary theory [1] for chains, equations (10) and (11) imply that

$$
R_{\lambda} e_{\mathbf{n}}(\theta)=\hat{R}_{\lambda}^{\min } e_{\theta}(\mathrm{n}) \text { for } \theta \neq 0,
$$

where $\left\{\hat{R}_{\lambda}^{\min }: \lambda>0\right\}$ is the resolvent of $\left\{\hat{T}_{t}^{\min }\right\}$. Hence

$$
T_{t} e_{\mathrm{n}}(\theta)=\hat{T}_{t}^{\mathrm{min}} e_{\theta}(\mathbf{n}) \quad(\forall t, \mathbf{n}) \text { for } \boldsymbol{\theta} \neq \mathbf{0} .
$$

Because $\left\{T_{t}\right\}$ is Feller, we can let $\theta \rightarrow 0$ in (13) and deduce that (13) also

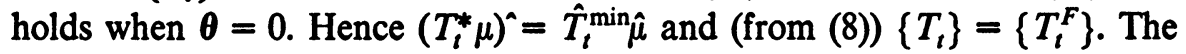
proof of Theorem 1 is complete. It should be noted that most of the proof is anticipated in [4].

3. Proofs of Theorems 2 and 3.

3.1. Recall that $\left\{\theta^{F}(t)\right\}$ denotes a diffusion process with transition semigroup $\left\{T_{t}^{F}\right\}$.

LEMMA 1. If the point 0 is not absorbing for $\left\{\boldsymbol{\theta}^{F}(t)\right\}$, then

$$
\sum_{\mathbf{n} \neq 0} G(\mathbf{0}, \mathbf{n})|\mathbf{n}|^{-2}<\infty
$$

where $G$ is the Green's function for the $\left\{p_{\mathrm{n}}\right\}$ random walk. 
Proof. If $\boldsymbol{O}$ is not absorbing for $\left\{\boldsymbol{\theta}^{F}(t)\right\}$, then (as remarked earlier) the strong Markov property implies that 0 is left immediately by $\left\{\theta^{F}(t)\right\}$. It follows easily that $R_{\lambda}^{F}(0, \cdot)$ is absolutely continuous with respect to the Haar measure. Hence, from (12) (extended to $\theta=0$ via the Feller property) and the Riemann-Lebesgue lemma,

$$
\begin{aligned}
\hat{R}_{\lambda}^{\min } 1(\mathrm{n}) & =\hat{R}_{\lambda}^{\min } e_{0}(\mathrm{n})=R_{\lambda}^{F} e_{\mathrm{n}}(0) \\
& =\int e_{\mathrm{n}}(\theta) R_{\lambda}^{F}(0, d \theta) \rightarrow 0 \quad \text { as } \mathrm{n} \rightarrow \infty ;
\end{aligned}
$$

in other words,

$$
x_{\lambda}(\mathbf{n}) \rightarrow 1 \quad(\mathbf{n} \rightarrow \infty), \quad \text { where } x_{\lambda} \equiv 1-\lambda \hat{R}_{\lambda}^{\min } 1 .
$$

Note that $0<x_{\lambda}<1$ and that, since 0 is absorbing, $x_{\lambda}(0)=0$. By Feller's theory of chains, $(\lambda-\hat{\mathfrak{L}}) x_{\lambda}=0$. Hence

$$
\mathcal{Q}\left(1-x_{\lambda}\right)=-\lambda \eta_{\lambda} \text {, }
$$

where

$$
\begin{aligned}
\eta_{\lambda}(\mathbf{m}) & \equiv|\mathbf{m}|^{-2} x_{\lambda}(\mathbf{m}) & & (\mathbf{m} \neq 0), \\
& \equiv \lambda^{-1} \sum p_{\mathbf{n}} x_{\lambda}(\mathbf{n}) & & (\mathbf{m}=\mathbf{0}) .
\end{aligned}
$$

[Digression. In the 1-dimensional case, the function $\eta_{\lambda}$ plays an important role as the Laplace transform of an entrance law for $\left\{\hat{T}_{t}^{\text {min }}\right\}$. See [4].]

Equation (16) shows that the function $1-x_{\lambda}$ is excessive for $\mathbb{Q}$. Hence, by the Riesz decomposition theorem (Spitzer [5]),

$$
1-x_{\lambda}=\lambda G \eta_{\lambda}+u_{\lambda}
$$

where $u_{\lambda}$ is $\mathbb{Q}$-harmonic. (Actually, since $u_{\lambda}$ is bounded harmonic, $u_{\lambda}$ is constant by the Choquet-Deny theorem, and since $u_{\lambda}(\mathbf{n}) \rightarrow 0$ as $\mathbf{n} \rightarrow \infty$ (because of (15)), we have $u_{\lambda}=0$.) From (15) and (17), $\eta_{\lambda}(\mathrm{m}) \sim|\mathrm{m}|^{-2}$ as $\mathbf{m} \rightarrow \infty$. We may therefore deduce (14) from the fact that $G \eta_{\lambda}(0)$ is finite.

3.2. Let

$$
\begin{aligned}
g(\theta) & \equiv|(\log |\theta|)|, & \text { if } d=2, \\
& \equiv|\theta|^{2-d}, & \text { if } d>3 .
\end{aligned}
$$

Recall that

$$
I \equiv \int g(\theta)[1-\varphi(\theta)]^{-1} d \theta .
$$

It is clear that we should work not with $g$ (which is appropriate for $\mathbf{R}^{d}$ ) and $I$ but with the appropriate modifications $(h$ and $J)$ for $T^{d}$. So let $q_{t}(\cdot, \cdot)$ denote the transition density function (with respect to the normalised Haar measure $d \theta$ ) of Brownian motion on $T^{d}$, and define 


$$
\begin{aligned}
h(\boldsymbol{\theta}) & \equiv \int_{[0, \infty)} e^{-1} q_{t}(\mathbf{0}, \boldsymbol{\theta}) d t, \\
J & \equiv \int_{T^{d}} h(\boldsymbol{\theta})[1-\varphi(\boldsymbol{\theta})]^{-1} d \boldsymbol{\theta} .
\end{aligned}
$$

The use of $J$ is equivalent to that of $I$ because $I=\infty$ if and only if $J=\infty$. This follows because there exist absolute constants $K_{d}$ in $(0, \infty)$ such that

$$
h(\theta) \sim K_{d} g(\theta) \text { as } \theta \rightarrow \mathbf{0} .
$$

(Transfer the classical asymptotic formulae from $\mathbf{R}^{d}$ to $T^{d}$ by covering.)

LEMMA 2.

$$
J=\sum_{\mathbf{n}} G(\mathbf{0}, \mathbf{n})\left[1+\frac{1}{2}|\mathbf{n}|^{2}\right]^{-1} \leqslant \infty
$$

so that $J<\infty$ if and only if (14) holds.

ProOF. Since $\frac{1}{2} \Delta e_{\mathrm{n}}(\theta)=-\frac{1}{2}|\mathbf{n}|^{2} e_{\mathbf{n}}(\theta)$, it is clear that

$$
\hat{h}(\mathbf{n}) \equiv \int e_{\mathbf{n}}(\theta) h(\theta) d \theta=\left[1+\frac{1}{2}|\mathbf{n}|^{2}\right]^{-1} \text {. }
$$

Formally, Lemma 2 is therefore just Parseval's theorem, but we have to be a little careful with the rigour.

For $0<r<1$, put

$$
G_{r}(0, \mathrm{n}) \equiv \sum_{k>0} r^{k} P_{k}(0, \mathrm{n})=\int e^{-i \mathrm{n} \cdot \theta}[1-r \varphi(\theta)]^{-1} d \theta,
$$

where $P_{k}(\mathbf{0}, \mathbf{n})$ denotes the $k$-step transition probability from $\mathbf{0}$ to $\mathbf{n}$ for the $\left\{p_{\mathrm{n}}\right\}$ random walk. We see that $[1-r \varphi(\theta)]^{-1}$ has Fourier coefficients in $\ell_{1}\left(Z^{d}\right)$. From this fact, it is easy to deduce that

$$
\int h(\theta)[1-r \varphi(\theta)]^{-1} d \theta=\sum_{\mathbf{n}} G_{r}(\mathbf{0}, \mathbf{n}) \hat{h}(\mathbf{n}) .
$$

(Recall that $G_{r}(\mathbf{0}, \cdot)$ and $\hat{h}(\cdot)$ are both symmetric in $\mathbf{n}$.) Now let $r \uparrow 1$ and note that $[1-r \varphi(\theta)]^{-1}$ converges monotonically to $[1-\varphi(\theta)]^{-1}$ on the "awkward" set on which $\varphi(\theta)>0$. After trivial adjustments, an application of the monotone-convergence theorem now completes the proof of Lemma 2.

3.3. In view of Lemmas 1 and 2, Theorem 2 will follow once we establish

LEMMA 3. If $J<\infty$, then 0 is not absorbing for $\left\{\boldsymbol{\theta}^{F}(t)\right\}$.

Proof. Let B be a Brownian motion on $T^{d}$ and set

$$
\tau(t) \equiv \frac{1}{2} \int_{0}^{t}[1-\varphi \circ \mathbf{B}(s)]^{-1} d s .
$$

The assumption that $J<\infty$ implies that $E_{0} \tau(\xi)<\infty$, where $\xi$ is an exponentially distributed random variable of rate 1 independent of $\mathbf{B}$. Thus $\tau$ 
is a bona fide continuous additive functional. Put

$$
\gamma(t) \equiv \inf \{s: \tau(s)>t\} .
$$

Then $\{\boldsymbol{\theta}(t)\} \equiv\{\mathbf{B} \circ \gamma(t)\}$ is a diffusion on $T^{d}$ which leaves $\mathbf{0}$ immediately.

For $f \in C^{2}\left(T^{d}\right)$,

$$
f \circ \mathbf{B}(t)-f \circ \mathbf{B}(0)-\int_{0}^{t} \frac{1}{2} \Delta f \circ \mathbf{B}(s) d s
$$

is a martingale (for every starting position). Since $\gamma(t) \leqslant 4 t$, the optionalsampling theorem implies that

$$
f \circ \mathbf{B} \circ \gamma(t)-f \circ \mathbf{B} \circ \gamma(0)-\int_{0}^{\gamma(t)} \frac{1}{2} \Delta f \circ \mathbf{B}(s) d s
$$

is a martingale. However, the expression at (20) is exactly

$$
f \circ \boldsymbol{\theta}(t)-f \circ \boldsymbol{\theta}(0)-\int_{0}^{t} \mathcal{L} f \circ \boldsymbol{\theta}(s) d s .
$$

Hence, if $\left\{T_{t}\right\}$ (acting on the space of bounded Borel functions on $T^{d}$ ) is the transition semigroup of $\{\boldsymbol{\theta}(t)\}$, then

$$
T, f-f=\int_{0}^{t} T_{s} L f d s \quad\left(\forall f \in C^{2}\left(T^{d}\right)\right) .
$$

Because of the singularity of $\mathcal{L}$ at $\mathbf{0}$, the Hung-Lamperti-Yang weak-convergence method [8] fails to establish that $\left\{T_{t}\right\}$ has the Feller property. However, the dual-process method succeeds as follows.

Since $\{\theta(t)\}$ leaves 0 immediately, it is straightforward to prove that the resolvent kernel $R_{\lambda}(\theta, \cdot)$ of $\{\theta(t)\}$ has the absolute continuity property for every $\theta$ in $T^{d}$. From this fact and (21), we can prove by the "RiemannLebesgue" argument in 2.2 that

$$
T_{t} e_{\mathrm{n}}(\theta)=\hat{T}_{t}^{\min } e_{\theta}(\mathrm{n})
$$

for all $\mathbf{n}$ and $\theta$ (including $\theta=0$ ). Hence $\left\{T_{t}\right\}=\left\{T_{t}^{F}\right\}$ as required.

The proof of Theorem 2 is now complete.

3.4. There is no need to write out a proof of Theorem 3. We know that the condition " $I<\infty$ " is necessary and sufficient for 0 to be an entrance boundary point for the unique Markov semigroup on $T^{d} \backslash\{0\}$ with generator extending $\mathcal{L}$. Theorem 3 merely translates this fact into different language.

For the Example mentioned after the statement of Theorem 3, take $d=2$ and, for $k=1,2$, introduce the function $\varphi_{k}$ on $T^{2}$ as follows:

$$
\varphi_{k}(\theta) \equiv 1-\left[1-\cos \theta_{k}\right]^{\alpha_{k}}=\sum_{r>1} c_{k, r}\left(\cos \theta_{k}\right)^{r}
$$

where

$$
c_{k, r} \equiv(-1)^{r+1}\left(\begin{array}{c}
\alpha_{k} \\
r
\end{array}\right)
$$


The condition $0<\alpha_{k} \leqslant 1$ implies that $c_{k, r} \geqslant 0$, so that $\varphi_{k}$ is the characteristic function of a symmetric distribution (a kind of "discrete stable symmetric distribution of index $2 \alpha_{k}$ ") concentrated on the $k$ th axis. Define $\varphi(\theta)$ as the weighted mean:

$$
\varphi_{k}(\theta) \equiv\left(2^{\alpha_{1}}+2^{\alpha_{2}}\right)^{-1}\left[2^{\alpha_{1}} \varphi_{1}(\theta)+2^{\alpha_{2}} \varphi_{2}(\theta)\right] .
$$

Then, as $\boldsymbol{\theta} \rightarrow \mathbf{0}$,

$$
1-\varphi(\theta) \sim\left(2^{\alpha_{1}}+2^{\alpha_{2}}\right)^{-1}\left[\left|\theta_{1}\right|^{2 \alpha_{1}}+\left|\theta_{2}\right|^{2 \alpha_{2}}\right],
$$

so that in a neighbourhood of $\mathbf{0}$, one may switch from a diffusion with generator extending $\mathcal{L}$ to a diffusion with generator extending $\frac{1}{2}\left[\left|\theta_{1}\right|^{2 \alpha_{1}}+\right.$ $\left.\left|\theta_{2}\right|^{2 \alpha_{2}}\right] \Delta$ via a time-transformation which is bounded on both sides by positive multiples of $t$. All that is required therefore is to check that $\int \log |\theta| d \theta /\left(\left|\theta_{1}\right|^{2 \alpha_{1}}+\left|\theta_{2}\right|^{2 \alpha_{2}}\right)$ is infinite if and only if $\alpha_{1}=\alpha_{2}=1$.

\section{BIBLIOGRAPHY}

1. W. Feller, On boundaries and lateral conditions for the Kolmogorov differential equations, Ann. of Math. (2) 65 (1957), 527-570. MR 19, 892.

2. I. V. Girsanov, An example of non-uniqueness of the solution of the stochastic equation of $K$. Itô, Teor. Verojatnost. i Primenen 7 (1962), 336-342 = Theor. Probability Appl. 7 (1962), 325-331.

3. R. Holley and D. Stroock, Dual processes and their application to infinite interacting systems, Advances in Math. (to appear).

4. R. Holley, D. Stroock and D. Williams, Applications of dual processes to diffusion theory, Proc. Sympos. Pure Math., vol. 31, Amer. Math. Soc., Providence, R. I., 1977.

5. F. Spitzer, Principles of random walk, Van Nostrand, Princeton, N. J., 1964. MR 30 \# 1521.

6. D. W. Stroock and S. R. S. Varadhan, Diffusion processes with continuous coefficients. I, II, Comm. Pure Appl. Maith. 22 (1969), 345-400; ibid. 22 (1969), 479-530. MR 40 \#6641; \#8130.

7. T. Yamada and S. Watanabe, On the uniqueness of solutions of stochastic differential equations. I, II, J. Math. Kyoto. Univ. 11 (1971), 155-167; ibid. 11 (1971), 553-563.

8. W. Zh. Yang, On the uniqueness of diffusions, $\mathrm{Z}$. Wahrscheinlichkeitstheorie und Verw. Gebiete 24 (1972), 247-261. MR 48 \#7401.

Department of Pure Mathematics, University College of Swansea, Singleton Park, SWANSEA SA2 8PP, Wales, Great Brttain 